\title{
SUL CONCETTO DI «IMPRENDITORE DI POLICY»
}

\author{
di Marco Giuliani
}

\section{Introduzione}

La diffusione di alcuni concetti nelle scienze sociali segue a volte percorsi imperscrutabili. Anche quando la sua origine non si perde in tempi lontani al punto da rendere necessaria una complessa ricostruzione etimologica, o quando il suo impiego non appare così strettamente improntato ad un contesto linguistico specifico, ripercorrere passo dopo passo la propagazione di un dato termine e il suo lento permeare il discorso scientifico può costituire un interessante percorso di ricerca.

In questa nota intendiamo approfondire analiticamente il concetto di policy entrepreneur, solitamente tradotto in italiano come «imprenditore di policy», rintracciandone l'origine e gli impliciti richiami teoretici, seguendone l'utilizzo nella letteratura politologica dedicata allo studio delle politiche pubbliche, dipanando $i$ possibili significati ad esso attribuiti, e chiarendo $i$ requisiti per un suo utilizzo nella ricerca empirica.

Si possono individuare due elementi che rendono opportuno, se non proprio necessario, tale compito. In primo luogo, sfogliando la letteratura specializzata ci si può facilmente accorgere di come tale concetto venga utilizzato sempre più di frequente $^{1}$. Non si tratta di una vera e propria moda - come forse

L'occasione per ripensare al concetto di imprenditore di policy è stata la partecipazione ad un progetto di ricerca finanziato dall'Unione europea e intitolato «Public Participation and Environmental Science E Technology Policy Options». Oltre ai partecipanti alla ricerca stessa, ringrazio Maurizio Ferrera e Claudio Radaelli per $i$ commenti alla prima versione di questa nota.

1 Già alla fine degli anni ottanta vi era chi parlava di «era dell'imprenditore di policy» (Beam, cit. in Weissert 1991). L'ondata non sembra comunque diminuire se, da una rapida ricerca su due dei repertori più comuni - «ABC PolSci» e «SocioFile» - si individuano dal 1990 al 1996 ben 16 articoli esplicitamente dedicati al medesimo tema, 
si potrebbe sostenere per concetti ancora più densi di significato, come quelli di governance o di network. Né siamo in presenza dei segnali di un diffuso cambiamento paradigmatico - come invece è avvenuto per i più tipici vocaboli dell'approccio neoistituzionale. Tuttavia si tratta di una crescita costante, che investe svariati settori di intervento (ambiente, sanità, governo urbano, istruzione, politiche economiche), e coinvolge soggetti decisionali diversi (legislatori, esperti, burocrati, leader politici, associazioni).

Non è difficile argomentare che parte di tale sviluppo sia dovuto ad una sorta di contaminázione teoretica, ad una graduale osmosi analitica che, con la tipica crescita geometrica dei contagi, finisce per propagarsi anche in luoghi e tempi assai distanti dal concepimento originario. Come si può ben immaginare, questa forma di diffusione non è priva di costi. Essa è infatti tanto più rapida ed efficace quanto più il concetto si presta ad adattamenti e manipolazioni, perdendo per strada i suoi connotati iniziali e finendo per divenire un passe-partout analitico di incerta collocazione. In questo, il fatto che il carattere imprenditoriale non sia tipico di una particolare categoria di attori ${ }^{2}$, ma qualifichi piuttosto un'operato, non aiuta di certo.

Se il successo del concetto di imprenditore di policy va in parte attribuito a ragioni endogene, cioè alla stessa logica dello sviluppo scientifico, non si può disconoscere che la sua affermazione sia anche legata ad un'effettiva ascesa di attori capaci di travalicare le tradizionali divisioni dei compiti nei processi di policy-making. È innegabile che, col tempo, la produzione di politiche pubbliche sia divenuta un fenomeno sempre più complesso: nuovi problemi sono stati posti all'attenzione della mano pubblica, nuovi livelli decisionali hanno reso i processi decisio-

mentre «Social Science Citation Index» ne riporta altri 6 nei primi dieci mesi del 1997 (per non contare i saggi all'interno di volumi, o quanto emerge con chiavi di ricerca quali «public» 0 «political entrepreneur»).

2 Si può anzi sostenere che sia proprio la loro eterogenea caratterizzazione a colpire maggiormente: gli imprenditori di policy non godono necessariamente di una legittimità formale, ma questo non impedisce loro di giocare un ruolo chiave nella produzione di politiche pubbliche; solo raramente si tratta di politici di professione, ma non per questo non sono impegnati a definire strategie per le decisioni collettivizzate; non è certo il modello burocratico legale-razionale ad improntarne l'azione, ma ciò non li esime dall'essere coinvolti anche nelle fasi di implementazione dei provvedimenti che auspicano; sono sicuramente esperti del settore in cui agiscono, ma questo non li deve necessariamente assimilare ai numerosi consulenti di cui le amministrazioni pubbliche spesso si avvalgono in assenza delle necessarie competenze interne. 
nali ancora più intricati, nuove competenze sono apparse necessarie per fornire risposte a richieste provenienti da cittadini e interessi variamente organizzati. Governare la democrazia non è probabilmente mai stato un mero esercizio di algebra delle preferenze (March e Olsen 1997), e la necessità di attori capaci di elaborare in modo innovativo soluzioni per problemi complessi, di districarsi fra le sedimentazioni storiche di passati interventi e nuovi vincoli all'azione pubblica, di gestire le relazioni interorganizzative, e di rendere coerenti esigenze solitamente contrapposte si è fatta sempre più pressante. La letteratura si è quindi occupata dei cosiddetti imprenditori di policy non solo per motivazioni auto-referenziali, ma perché essi, di fatto, sono divenuti via via più presenti e visibili nei processi di produzione di decisioni collettivizzate.

In secondo luogo, proprio in virtù dell'apparente malleabilità dei contenuti analitici di tale concetto e della problematica definizione del rapporto significante-significato, la consistenza empirica della variabile «ruolo dell'imprenditore di policy» è assai incerta. Se, banalmente, si finisce per definire come imprenditoriale il ruolo giocato da qualsiasi attore che nel processo di policy-making assume un ruolo strategico, o riesce ad ottenere un qualche risultato di rilievo in condizioni avverse, allora non esiste forse processo decisionale senza un suo imprenditore, e non esiste attore politico che non possa sfruttare imprenditorialmente determinate congiunture.

$\grave{E}$ vero che il costituzionalismo empirico che fonda gli studi di policy impone di «partire dal basso», dal ruolo effettivamente esercitato nei processi decisionali più che dalle attribuzioni formali di legittimità, ma questo non significa attribuire statuto di imprenditorialità ad ogni azione capace di registrare un certo successo. In caso contrario saremmo costretti a definire «imprenditore di policy» un ministro capace di far costruire un'autostrada inutile per favorire gli interessi dei costruttori, oppure un politico che, negli scambi che avvengono all'interno delle commissioni parlamentari, riesce a ottenere un provvedimento per la propria circoscrizione elettorale, o ancora un burocrate che sfrutta la propria posizione strategica per dirottare risorse pubbliche o per favorire questo o quel destinatario ${ }^{3}$. In questo

$3 \mathrm{E}$ in questa direzione che vanno anche gli sforzi di Weissert (1991) quando distingue fra policy entrepreneurs e policy opportunists, o di Wallis e Dollery (1997) quando parlano di policy leaders e vote-buying politicians. 
caso, le tradizionali etichette di «governo spartitorio» e di «patronage» sembrano le più appropriate a definire tali pratiche, mentre una loro ricatalogazione in chiave di policy entrepreneurship non farebbe altro che offuscare le sottostanti logiche decisionali. Dal punto di vista empirico si ripresenta qui, specularmente, lo stesso problema che sussiste nell'individuazione concreta delle politiche simboliche e nella loro distinzione rispetto ai più comuni deficit di implementazione. Se è da un lato assodato che queste politiche sono caratterizzate dall'assenza di un qualsiasi impatto reale, giacché «soddisfano» le esigenze dei destinatari solo in modo fittizio, dall'altro è altrettanto evidente che la mancanza di risultati non può essere l'unico requisito di queste stesse politiche, pena l'attribuire ad originari intenti simbolici ogni fallimento di policy. Allo stesso modo, benché modalità imprenditoriali nel perseguimento di obiettivi di policy possano produrre esiti concreti anche in circostanze avverse, considerare il successo come l'inequivocabile cartina di tornasole della presenza di policy entrepreneurs è del tutto fuorviante: anche in questo caso, una più precisa definizione delle sue proprietà contribuirebbe a ridurre l'eccessiva e ambigua estensione del concetto.

La presente nota è organizzata nel modo seguente. Nel prossimo paragrafo cercheremo di identificare quali sono i caratteri costitutivi dell'azione imprenditoriale nei processi di produzione e realizzazione delle politiche pubbliche. Sulla base delle dimensioni analiticamente individuate, proporremo quindi una tipologia degli imprenditori di policy, per poi avanzare alcune ipotesi circa le risorse su cui tali attori possono contare, e le condizioni ambientali che ne favoriscono l'operato.

\section{Una definizione e una tipologia}

L'espressione «policy entrepreneun accosta due termini che solitamente competono a due universi semantici distinti. Il concetto di policy afferisce più o meno direttamente alla sfera dell'allocazione autoritativa di valori, alla produzione di decisioni collettivizzate, mentre quello di entrepreneur appartiene solitamente all'ambito della produzione economica, al libero dispiegarsi di interazioni competitive volte all'ottenimento di profitti. Le politiche sono tradizionalmente politiche pubbliche, quanto gli imprenditori sono solitamente imprenditori privati. Già in 
questa sorta di ossimoro sta un segno del carattere poco tradizionale di questo tipo di attore decisionale, della sua capacità di infrangere o ignorare consolidate linee di divisione.

Se partiamo dalla definizione che Schneider e Teske danno di «political entrepreneur», cioè di «individui che cambiano la direzione e il flusso della politica» $(1992,737)$, e, a parità di altre condizioni, sostituiamo la produzione di politiche pubbliche alla competizione per il potere, potremmo semplicemente dire che gli imprenditori di policy sono coloro che indirizzano e controllano il flusso delle politiche.

Come definizione iniziale parrebbe del tutto legittima, se non sollevasse due ordini di problemi. In primo luogo, nel passaggio dalla politica alle politiche cambia il rapporto che gli attori hanno con il potere. Per dirla con il linguaggio della game theory, il gioco della politica è a somma zero, non cooperativo: le logiche coalizionali che possono caratterizzarlo sono solo funzionali alla spartizione di poste inalterabili - la maggioranza parlamentare, il governo, la posizione di primo ministro. Vi è un'unica arena di confronto, che è quella appunto dove si compete per questo potere finito. Il gioco delle politiche non ruota invece attorno a benefici in misura finita. Uno dei suoi obiettivi, in effetti, è quello di stabilire l'entità e la forma stessa delle poste in gioco: regole, risorse, costi, trasferimenti. Le politiche, nella nota espressione di Heclo e Wildavsky (1981, XIV), «non sono delle costanti, ma delle variabili», ed ognuna di esse rappresenta l'occasione per aprire una nuova arena dove le relazioni di potere vengono a definirsi ${ }^{4}$.

Allo stesso modo, in un mondo di risorse di potere finite l'imprenditore politico è un soggetto al singolare, colui che appunto presidia l'accesso a tali risorse. In arene multiple, invece, in cui $\mathrm{i}$ processi decisionali «espandono il potere su ogni dimensione» (Heclo e Wildavsky 1981, XIV), tale ruolo è di fatto giocato da molteplici attori; potenzialmente da tutti. In questo senso, indirizzare la politica è diverso da indirizzare le politiche tanto quanto una situazione di monopolio è diversa da una di mercato. La pluralità degli attori in grado di influenzare il corso e il flusso del policy-making rendono la traduzione dell'origina-

4 Regonini (1995) parla a questo riguardo di potere al singolare e al plurale, sottolineando come il passaggio dal primo al secondo tipo di giochi costituisce una soluzione di continuità tale da avere notevoli ripercussioni sul tipo di relazioni che si instaurano fra gli attori politici. Rimandiamo a tale lavoro per approfondimenti su questo punto. 
ria definizione di Schneider e Teske poco proficua sotto il profilo analitico.

$\mathrm{Vi}$ è un secondo aspetto, strettamente connesso con il primo, che amplifica la distanza fra le due sfere di azione - quella politica e quella di policy. Nel primo caso l'azione imprenditoriale sembra poter essere giocata esclusivamente da un soggetto decisionale, per così dire, «in carne ed ossa». L'imprenditore politico è una persona, probabilmente dotata di risorse o qualità uniche: non è e non può essere una organizzazione, come un partito politico, né un'istituzione, come un ministero. Nella sfera del policy-making, invece, si è gradualmente diffusa un'accezione dell'azione imprenditoriale che coinvolge soggetti decisionali impersonali. La Commissione europea, ad esempio, è stata tratteggiata come imprenditore di policy nei confronti degli attori nazionali e delle altre istituzioni comunitarie (Laffan 1997), e lo stesso può essere detto - oltre che per singoli policy-makers - per una commissione parlamentare particolarmente attiva e capace, per una comunità epistemica coesa ed efficace, per una think tank centrale nel dibattito attorno ad una politica pubblica, e così via ${ }^{5}$. Insomma, se gli attributi che qualificano il concetto di political entrepreneur vengono semplicemente trasferiti su quello di policy entrepreneur, l'estensione di quest'ultimo si fa eccessiva, rendendo per questo necessaria l'individuazione di proprietà aggiuntive.

In questa direzione non ci è di molto aiuto la definizione data da Kingdon $(1984,214)$, che pure è fra gli studiosi che in modo più sistematico ha inserito l'azione degli imprenditori di policy all'interno di un completo schema interpretativo. Essi sarebbero infatti «persone disposte ad investire risorse di tipo diverso nell'aspettativa di un ritorno futuro sotto forma di politiche auspicate». Ma quali sono gli attori decisionali che non fanno proprio questo? A questa stregua, nuovamente, tutti i policymakers avrebbero carattere imprenditoriale. Una qualità che invece potrebbe discriminarne l'operato è il grado di innovazione che essi apportano ai processi decisionali (Mintrom 1997; Ro-

5 Va da sé che questo non comporta un'«antropomorfizzazione» delle istituzioni, quanto il riconoscere che l'imprenditorialità permea a tal punto la cultura organizzativa da improntare di sé l'azione dei soggetti che in essa agiscono: la Commissione europea, ad esempio, può essere considerata imprenditore di policy perché la sua storia, il suo ruolo e la sua costituzione materiale spingono i suoi funzionari a non adattarsi a modelli tradizionali di amministrazione incentivandone comportamenti innovativi (si veda sul punto anche Nugent 1995 e Radaelli 1998). 
berts 1992). Si può anzi sostenere che «l'imprenditore di policy può essere definito un catalizzatore [della...] innovazione nel campo delle politiche pubbliche» (Maraffi 1996, 188; ripreso da King e Roberts 1987), colui che attiva e rende possibile il cambiamento di policy. In questo, nel suo essere al contempo leader e innovatore, egli si avvicina al modello di imprenditorialità tratteggiato da Schumpeter, ricalcandone sostanzialmente gli attributi in una diversa sfera dell'agire sociale.

Di fatto, qualunque sia il grado di trasformazione che il suo intervento riformatore comporta - la modifica degli strumenti adottati piuttosto che un vero e proprio cambiamento di paradigma - il mutamento può avvenire grazie a due diverse modalità d'azione: da un lato, sul piano cognitivo, l'imprenditore di policy cerca di manipolare il cosiddetto discorso di policy, cioè l'insieme di idee e argomenti che sostanzia la pretesa di risoluzione dei problemi collettivi; dall'altro, sotto il profilo strategico, egli tenta di modellare reti di relazioni e coalizioni decisionali originariamente inesistenti. Cerchiamo ora di approfondire queste due dimensioni analitiche.

Le politiche pubbliche non sono fenomeni che si autodefiniscono. Su questo punto sono d'accordo tutti gli studiosi, anche se esistono modi più o meno radicali per riaffermare tale posizione. Questo vuole dire che una delle prime domande a cui deve rispondere sia chi studia una politica pubblica, sia chi cerca di costruirla, è semplicemente: «che problema ho di fronte?», oppure, per dirla di nuovo con March e Olsen (1997, 15), «in che tipo di situazione mi trovo?». Le situazioni, i contesti, i problemi di fondo non sono a loro volta auto-evidenti: non esiste dato che non necessiti di interpretazione (Stone 1988), e non esiste evidenza che non sia sostenuta da un argomentare (Majone 1989). È questo uno dei motivi che rende gli aspetti cognitivi dei processi decisionali tanto rilevanti. In caso contrario, in assenza di ambiguità e di margini di valutazione, non vi sarebbe che spazio per la bilancia dei poteri, mentre proprio il supremo dei poteri - la definizione delle alternative (Schattschneider 1960) - verrebbe disinnescato: gli attori decisionali non potrebbero «interrogarsi», non rimanendo loro che «contarsi» (per provare - come già molti hanno fatto - a rendere in italiano la dicotomia puzzle-power di Heclo 1974).

Sotto questo profilo, l'imprenditore di policy è colui che innova non nelle tecniche o nell'equilibrio delle risorse, ma nell'interpretazione della situazione. Egli non scopre tanto nuove 
soluzioni per vecchi problemi, ma riformula i problemi stessi. Le controversie di policy più intrattabili non vengono sciolte da un taglio netto, ma da una ridefinizione del quadro interpretativo, da ciò che Schön e Rein (1994) definiscono come re-framing. In quest'accezione, l'eresteta di Riker (1983) è forse il soggetto decisionale che maggiormente sfrutta a suo favore la ridefinizione del discorso di policy (White 1994), ma lo stesso si può dire per tutti quei policy-makers che agiscono più sul piano cognitivo che su quello strategico, indipendentemente dal fatto che ne traggano benefici personali o meno. Le idee di cui si fanno portatori e interpreti - Milward (1980) riprende a questo riguardo Schön (1971) e parla degli imprenditori di policy come promotori di «ideas in good currency» - sono quindi volte ad estendere la gamma delle compatibilità, a rivelare nicchie d'azione inesplorate, a creare nuove opportunità e giochi a somma positiva ${ }^{6}$ : la riformulazione di problemi tradizionali mira, ad esempio, a coniugare tutela dei lavoratori e flessibilità, standard ambientali e profitto economico, universalismo e selettività nella protezione del welfare, consenso ed efficienza decisionale, garanzia per i cittadini e certezza per l'amministrazione.

$\mathrm{Ma}$ veniamo alla seconda dimensione che caratterizza l'innovazione prodotta dagli imprenditori di policy, quella che potremmo definire di tipo «strategico». Molti studiosi hanno avanzato l'idea che la definizione del contenuto dell'intervento pubblico, così come le sue stesse dinamiche, siano controllati $\mathrm{da}$ reti di attori variamente qualificate ma, comunque, di tipo segmentato. Ogni settore di policy, e forse addirittura ogni singola politica pubblica vedrebbe un insieme definito di soggetti decisionali capaci di orientarne la filosofia, controllarne gli aspetti simbolici, manipolarne le risorse, indirizzarne l'evoluzione e così via. Queste reti di attori possono essere più o meno coese e stabili, più o meno aperte a nuovi ingressi, più o meno consensuali e caratterizzate da valori comuni, e per specificare

6 In questo senso, il filtro cognitivo degli imprenditori di policy sembrerebbe creare punti di equilibrio laddove questi non parrebbero esistere. Lo stesso attore potrebbe però avere un ruolo anche nell'opposta situazione di instabilità, laddove cioè l'esistenza di molteplici equilibri favorirebbe la circolarità degli esiti. Secondo Garrett e Weingast (1993), ad esempio, la Corte di giustizia europea ha ridotto la gamma dei possibili equilibri nell'instaurazione del cosiddetto Mercato interno, riuscendo ad imporre l'idea di mutuo riconoscimento e facendo di essa uno dei cardini del processo d'integrazione. In questo caso, nella terminologia dell'analisi razionale, l'imprenditore di policy potrebbe essere colui che risolve i dilemmi dovuti all'incompletezza dei contratti. 
questa loro diversa caratterizzazione si fa riferimento a termini diversi, quali «sottosistemi», «comunità», networks, advocacy coalitions, ecc. (Capano e Giuliani 1996).

Ebbene, i policy entrepreneurs tendono ad attraversare $\mathrm{i}$ confini - robusti o tenui - di queste suddivisioni per modellare reti più ampie, per connettere attori isolati o privi di voce, per interpretare le posizioni e le preferenze di interessi diffusi. Essi mirano a costruire nuove coalizioni a supporto di un progetto di policy, soprattutto là dove queste sono impedite dai limiti intrinseci alla mobilitazione collettiva (Schneider e Teske 1992; Mintrom e Vergari 1996). È a questo tipo di operazione che si riferiva Wilson (1980) nella sua tipologizzazione delle politiche regolative quando identificava la casella dell'azione «imprenditoriale» come quella che portava alla produzione di benefici diffusi a fronte di costi concentrati. Ed è per questo che gli imprenditori di policy spendono così tanto tempo ad intrecciare relazioni: «networking in and around», come dice Mintrom (1997, 739).

In realtà, questa attività di collegamento può essere sia esplicitamente ricercata, che avvenire, per così dire, «inconsciamente», fornendo i mezzi per il mutuo riconoscimento, per la costruzione dei sufficienti livelli di fiducia ed il reciproco scambio di informazioni fra gruppi di attori altrimenti debolmente connessi. L'imprenditore diviene lo snodo implicito, il traduttore di linguaggi ed esperienze originariamente diverse, colui che costruisce quelle che vengono definite discourse coalitions (Hajer 1995), cioè reti di attori che non si incontrano fisicamente, ma che si riconoscono in una comune «narrazione» del problema di policy (Roe 1994), in una specifica story-line. Questa osservazione rappresenta anche il punto di incontro fra le due dimensioni dell'azione imprenditoriale qui individuate: le idee, le mappe concettuali, gli scenari non sono solo il substrato intellettuale della produzione di politiche o il supporto per una giustificazione pubblica delle scelte di policy, ma divengono anche il collante per la costruzione strategica di alleanze, il mezzo per ridurre i costi di transazione e favorire la mobilitazione collettiva.

Ridefinizione del discorso di policy e superamento dei consolidati cleavages coalizionali sembrano dunque essere le due modalità che sostanziano l'azione innovativa degli imprenditori di policy: queste strade non devono necessariamente essere perseguite entrambe nello stesso tempo, anche se abbiamo già avu- 
to modo di notare come non solo l'una non escluda l'altra, ma come esse possano intrecciarsi in modo imprevisto e sostanzialmente non ricercato. Se noi incrociamo tale dimensione con l'individuazione del tipo prevalente di legittimazione dell'attore imprenditoriale - una legittimazione formale, tramite un'investitura pubblica, piuttosto che una legittimazione informale, di un attore privato, dovuta alle personali competenze e capacità - otteniamo una tipologia dei policy entrepreneurs che può ulteriormente chiarire le caratteristiche del concetto qui trattato ${ }^{7}$.

Le etichette attribuite a ciascuna casella possono anche essere considerate arbitrarie, ma esse da un lato rendono abbastanza bene l'idea di come individualmente si configuri l'azione di tali policy-makers, e dall'altro risultano sostanzialmente coerenti con l'utilizzo consolidato di tali termini in letteratura. Un attore pubblico che mira ad estendere i networks in cui è coinvolto per la produzione di politiche pubbliche svolge un'azione di leadership all'interno di quelle reti, come è avvenuto, ad esempio, con il ministro Ruffolo nel momento della istituzionalizzazione del ministero dell'Ambiente e dei nuovi rapporti instaurati con le associazioni ambientaliste. Ruffolo stesso, una volta terminata la sua esperienza nell'esecutivo, e quindi dimessi i panni formali, ha comunque mantenuto per un certo tempo un ruolo strategico nel far comunicare attori istituzionali, imprenditori e gruppi d'interesse pubblico, svolgendo quindi importanti compiti di brokerage. Fra quelli qui utilizzati, il termine di broker è forse quello che ha avuto maggiore fortuna analitica, quanto meno nella letteratura dedicata all'indagine delle strutture delle reti di relazione sociale (Gould e Fernandez 1989; Fernandez e Gould 1994). Noi lo utilizziamo qui in una accezione abbastanza minimalista come soggetto (privato) che facilita lo scambio e le interazioni fra gruppi di attori o livelli decisionali normalmente poco comunicanti (Marsden 1982).

Sul piano cognitivo, invece, va considerato come innovatore quell'attore decisionale pubblico che reinterpreta il proprio ruo-

7 Non è necessario che i singoli casi reali possano venire attribuiti inequivocabilmente ad un'unica cella della tipologia, giacché stiamo qui compiendo un esercizio di scomposizione concettuale e non di investigazione empirica: in questo senso, il criterio della esaustività è senz'altro più rilevante di quello della mutua esclusione. In ogni caso, anche sotto questo secondo profilo si potrebbe argomentare che i singoli imprenditori di policy posseggano determinate caratteristiche in misura prevalente rispetto ad altre: che, ad esempio, agiscano più sul piano strategico che su quello cognitivo, risolvendo in questo modo l'incertezza riguardo all'attribuzione tipologica. 


\begin{tabular}{llcc}
\hline \multirow{2}{*}{ Legittimazione } & \multicolumn{2}{c}{ Attività } \\
\cline { 3 - 4 } & $\begin{array}{l}\text { Formale / } \\
\text { Pubblico }\end{array}$ & Strategica \\
costruzione di reti & $\begin{array}{c}\text { Cognitiva } \\
\text { promozione di idee }\end{array}$ \\
\cline { 2 - 4 } & $\begin{array}{l}\text { Leader } \\
\text { Privato }\end{array}$ & Innovatore \\
\hline
\end{tabular}

FIG. 1. Una tipologia degli imprenditori di policy.

lo in funzione di un progetto: a livello internazionale, ad esempio, si potrebbe sostenere che Delors, nella sua qualità di presidente della Commissione delle Comunità europee, abbia rappresentato al meglio questa specifica modalità pubblica di azione imprenditoriale $e^{8}$. Su un piano interno, invece, e benché si possa ritenere che le soluzioni proposte non siano né del tutto originali, né prive di legami con precedenti illuminati tentativi', la qualifica di policy entrepreneur innovatore potrebbe forse essere assegnata a Bassanini, se non altro per una certa capacità di disinnescare consolidate opposizioni alle riforme della pubblica amministrazione. Infine, il termine promotore è forse quello meno impegnativo sotto il profilo valoriale, ma sostanzialmente individua singole persone (o organizzazioni) capaci di illuminare di luce nuova vecchie questioni, fornendo una reinterpretazione anche globale del nesso problema-soluzione. Parte della letteratura politologica si è preoccupata di ricostruire le biografie di

8 A questo riguardo, Dyson e Featherstone (1997) analizzano il ruolo esercitato da Delors nell'impostare il progetto di Unione economica e monetaria, e mettono bene in luce sia la dimensione strategica che quella cognitiva del suo agire: «Delors era abile nel mettere in cantiere idee, nel connettere persone e nel creare le circostanze adatte» (Intervista in ibidem). Gli autori, a dire il vero, utilizzando una distinzione nelle funzioni svolte dalla Commissione europea avanzata da Ludlow (1991), contrastano i comportamenti da «strateghi» e quelli da «animatori» caratterizzanti la fase di agenda setting, con quelli più tipicamente imprenditoriali che governerebbero la formulazione delle politiche in senso stretto. In questo senso, Delors avrebbe mostrato un maggior controllo sugli elementi simbolici, cognitivi e coalizionali nella prima fase del processo relativo l'Uem, perdendone in parte le fila successivamente all'inaugurazione della Conferenza intergovernativa.

$9 \mathrm{Ma}$, come dice Kingdon (1984), è imprenditore chi sfrutta la finestra d'opportunità per portare in porto quel progetto che ha a lungo galleggiato nella «zuppa primordiale» delle idee. 
tali personaggi, ma, per fornire esempi assai noti, ci vengono qui in mente operatori come Basaglia nel riformare l'approccio agli interventi sulla salute mentale, studiosi come Hirschman per quanto riguarda i progetti di sviluppo nel terzo mondo, e alcune associazioni e istituti di ricerca ambientalisti nel riorientare il dibattito mondiale in direzione dello sviluppo sostenibile.

Se fino a qui abbiamo cercato di tratteggiare la complessità interna di un concetto quale quello di imprenditore di policy, principalmente per allontanarci da un uso opportunistico dello stesso che lo vuole sinonimo di garante di imprevisti successi di policy, nel prossimo paragrafo intendiamo approfondire il tema delle risorse che permettono a tale attore di svolgere le sue particolari funzioni decisionali, e del contesto che ne favorisce o limita l'azione.

\section{Risorse, temi e contesti}

Non si possono concepire gli imprenditori di policy esclusivamente come personaggi dotati di carisma. Si finirebbe per relegare il loro ruolo nel campo della eccezionalità, mentre nelle pagine iniziali abbiamo individuato un nesso fra la crescente complessità dei processi decisionali e la diffusione di questo peculiare attore del policy-making. Se un policy entrepreneur è dotato di qualità carismatiche che ne facilitano la comunicazione e il potere persuasivo, questo non fa che agevolarne il compito, ma non si può ridurre né il contenuto della sua azione innovativa, né il tipo di legittimazione che richiama al possesso di virtù uniche.

$\mathrm{Ne}$ consegue che anche l'autorità dell'imprenditore di policy necessita di essere continuamente riaffermata e legittimata. In un certo senso, l'etero-riconoscimento del suo ruolo nel processo decisionale è forse ancora più essenziale di quello necessario ad altri partecipanti al processo di policy-making. Sia in senso strategico - per costituire ed essere lo snodo di un sistema di relazioni - che in senso cognitivo - per promuovere una diversa concettualizzazione del problema di policy e delle soluzioni plausibili - il policy entrepreneur è strettamente legato alla disposizione mostrata dagli altri attori decisionali nei suoi confronti. Il suo legittimarsi come catalizzatore dell'innovazione dipenderà quindi fortemente dal riconoscimento proveniente $\mathrm{da}$ sfere diverse dal suo normale campo d'azione: per un attore po- 
litico, ad esempio, sarà fondamentale acquisire il consenso degli apparati amministrativi e, contemporaneamente, saper parlare il linguaggio dell'economia; per un esperto che intende incidere direttamente nel policy-making, sarà importante la sua capacità di abbandonare le argomentazioni esoteriche della scienza per convincere da un lato il parlamentare desideroso di essere rieletto, e dall'altro l'associazione di interessi pubblici. Nessuna azione imprenditoriale può fare semplicemente conto sui tradizionali bacini di consenso e su modalità consuete per la sua acquisizione: è necessario saper agire contemporaneamente «su più tavoli» perché è solo dalla molteplicità delle fonti di legittimazione che l'imprenditore di policy ricava un'autorità qualitativamente diversa da quella degli altri attori decisionali. Per fare questo, egli utilizzerà risorse che in parte si sovrappongono e in parte si diversificano da quelle attivate da questi ultimi. «[Esse...] possono essere sia di tipo organizzativo che personali, [fra cui...] abilità intellettuali, conoscenza specifica del campo di intervento, propensione a dirigere o a costituire gruppi di lavoro, reputazione, contatti, capacità strategiche e tenacia» (Mintrom e Vergari 1996, 423-424). Proviamo ad analizzarle più puntualmente.

La prima risorsa che viene messa in evidenza dalla letteratura è il tempo. Ogni attore decisionale ha un rapporto peculiare con questa variabile. Ė risaputo che il politico, specie quello appartenente ad una compagine di governo, ha la necessità di ottenere risultati nel breve periodo, e comunque prima della successiva scadenza elettorale: la teoria del ciclo politico spiega con dovizia di analisi questo legame. Il burocrate - quantomeno nella sua stilizzazione popolare - sembra avere invece una predilezione per i tempi lunghi, quasi per l'eternità: anche allontanandosi da queste forme di semplificazione, non si può non riconoscere un legame fra il carattere permanente del suo ufficio e l'indifferenza per tutto ciò che è rapido, temporaneo, transitorio. Gli stessi gruppi di pressione, in particolare quelli che tutelano interessi diffusi, hanno un rapporto caratteristico con il tempo, soggetti come sono ai cicli di attenzione pubblica che, a parità di sforzi, ne amplificano o mortificano i risultati. Avere una scadenza obbligata può accelerare processi decisionali che apparivano definitivamente insabbiati, così che la fretta sembra costituire un'arma contro i policy-makers più recalcitranti. Al tempo stesso, la necessità di arrivare ad un risultato nel breve periodo permette proprio a questi ultimi di ottenere il massimo 
dei benefici grazie alla indisponibilità al rinvio dei loro avversari (Dente 1996).

Scomporre i legami esistenti e ricostruire nuove reti decisionali, così come promuovere un discorso di policy radicalmente diverso, sono operazioni che richiedono tempo. Per questo motivo il tempo è una risorsa necessaria agli imprenditori di policy, e la perseveranza o la costanza vengono considerate una delle qualità a loro più utili. Non ogni momento è appropriato per impegnarsi nel tessere relazioni fra attori tradizionalmente non comunicanti, così come anche le migliori idee necessitano di entrare in un dibattito nel momento più opportuno se si intende riorientarne il corso. Nell'immagine di Kingdon (1984), gli imprenditori di policy sono come surfisti che devono continuamente rimanere a galla in attesa dell'onda giusta, della finestra di opportunità che ne massimizzerà l'azione: se non presidiano la propria arena decisionale con attenzione, o se si lanciano troppo presto o troppo tardi, i loro sforzi saranno vani.

Un secondo tipo di risorsa che, di fatto, emerge dai diversi studi empirici è legata al ricoprire una qualche rilevante carica formale. Benché questa non sia una condizione necessaria per svolgere un'azione di tipo imprenditoriale, né si vuole qui sostenere che esistano incarichi di per sé capaci di attivare innovazione, il ruolo istituzionalmente ricoperto può rivelarsi una preziosa risorsa da attivare e sfruttare al momento opportuno. L'istituzione - sia essa pubblica o privata - conferisce una riserva di legittimazione, così che anche comportamenti «poco ortodossi», privi di giustificazioni tradizionali o legali-razionali, possano essere provvisoriamente accettati «a scatola chiusa». Un ministro dell'ambiente - ad esempio - può decidere di istituzionalizzare una prassi di consultazione con le associazioni ecologiste; la leadership di un sindacato può decidere di abbandonare la tutela del salario eguale per tutti a favore di uno scambio flessibilità-occupazione; il sindaco di una città può decidere di sperimentare le idee di «città senza auto» di cui ha sentito parlare in sede europea, e così via. La carica istituzionale costituisce ovviamente una risorsa per comportamenti diversi: per azioni nel solco della consuetudine così come per innovazioni, per interventi rivolti al bene pubblico (qualunque cosa possa essere intesa con questo termine) così come per interessi privati. Sfruttarla per una qualche forma di policy entrepreneurship è solo una delle possibilità, e nemmeno la più diffusa; soprattutto se si considera che le riserve di legittimazione che essa fornisce non 
sono affatto eterne, ampliando solo provvisoriamente i margini di libertà per l'attore di policy, che deve comunque fare i conti con le resistenze interne all'istituzione stessa.

Un terzo tipo di risorsa attivabile è rappresentata dai legami di tipo personale. Questa «carta», così come quella precedente, sembra essere maggiormente connessa ad azioni di tipo strategico, quelle che impegnano leader e brokers, piuttosto che a innovazioni sul piano cognitivo. Ogni policy-maker ha una sua storia personale, fatta delle competenze che ha acquisito durante la sua formazione, ma anche delle persone che ha frequentato, delle organizzazioni per cui ha lavorato, delle istituzioni a cui è stato affiliato o con cui è entrato in contatto. Questi rapporti possono risalire alla giovinezza, agli studi universitari, o dipendere semplicemente dal fatto che determinate coorti di persone sono cresciute nello stesso periodo in un medesimo contesto, partecipando agli stessi eventi ed essendo soggetti agli stessi influssi culturali. Ebbene, questi legami, talvolta anche deboli, possono acquisire rilevanza proprio al momento della ricostruzione di reti trasversali di attori, per la possibilità di instaurare relazioni dirette, di utilizzare un linguaggio ed uno stile comunicativo comuni, di far conto su un substrato di fiducia reciproca che facilita atteggiamenti cooperativi. I contatti di tipo personale, anche quelli non attivati da tempo, permettono una più rapida circolazione delle informazioni e, nel contempo, minimizzano le possibilità di fraintendimento e logiche da free rider $^{10}$.

Infine, un quarto tipo di risorsa, particolarmente importante sul versante delle innovazioni realizzate attraverso un riorientamento cognitivo del discorso di policy, è quella relativa al controllo selettivo delle informazioni e alla competenza specialistica. Benché il monopolio della conoscenza appaia come tipico di una particolare classe di attori - gli apparati tecnici interni all'amministrazione o gli esperti che fungono da consulenti per $\mathrm{i}$ decisori pubblici e privati - va riconosciuto che con il generalizzarsi del fenomeno dei cosiddetti «puri ibridi», cioè di attori che si muovono costantemente sul confine fra competenze di-

10 In Regonini e Giuliani (1994) si sostiene, ad esempio, che la comunità epistemica dei professori universitari che in Italia ruotava attorno ad arene di policy nevralgiche è stata particolarmente rilevante come agente di cambiamento proprio per la facilità del «mutuo riconoscimento» fra le sue componenti. Che l'attuale presidente dell'Enel abbia militato fra le fila ecologiste non può non avere implicazioni sulle relazioni che tale importante ente pubblico intrattiene con l'universo ambientalista, e potremmo proseguire a lungo con tale tipo di esemplificazioni. 
verse, è aumentata la gamma di soggetti decisionali che gode di un accesso non occasionale a dati e informazioni strategiche per il policy-making. Non è necessario che l'expertise richiesta faccia parte del bagaglio personale dell'attore decisionale, quanto che egli possa e sappia avere accesso alle necessarie fonti di produzione e diffusione di conoscenza, alle reti internazionali di validazione del sapere, alle think tanks dotate della necessaria reputazione (Stone 1998). Questa possibilità non è né patrimonio esclusivo delle istituzioni pubbliche, né necessariamente legata alla quantità relativa di risorse gestite all'interno di un determinato settore d'intervento. Gli imprenditori di policy si possono avvantaggiare di questa relativa democratizzazione dell'accesso alla conoscenza, favorendo la circolazione delle idee e facendosi promotori delle soluzioni più innovative. Essi possono connettere circuiti non comunicanti, incoraggiando la contaminazione fra soluzioni e promuovendo le suggestioni provenienti da ambiti diversi.

Queste quattro categorie di risorse potrebbero poi essere declinate più nello specifico al fine di riconoscere ulteriori fattori facilitanti l'operato dell'imprenditore di policy: un qualche tipo di investitura rappresentativa (proveniente dal basso o concessa dall'alto) facilita certamente il compito degli attori privati, così come una reputazione dovuta a precedenti esperienze di successo o un qualche riconoscimento internazionale non può che legittimarne il ruolo a fronte degli attori più scettici o inerziali. Tempo, margini istituzionali, contatti personali e accesso alle conoscenze, da soli, non «fanno» un policy entrepreneur, ma chiunque si proponesse di essere catalizzatore di innovazione all'interno di uno specifico settore d'intervento senza possedere almeno alcune di tali risorse, perderebbe ben presto il riconoscimento e la legittimazione necessari.

$\mathrm{Vi}$ sono ancora due questioni che vorremmo brevemente toccare in questa nota, e cioè se, pur all'interno di una tendenza generale alla diffusione di questo tipo di attori, vi siano tematiche e contesti istituzionali o ambientali che favoriscono l'emergere e l'operato degli imprenditori di policy, ed altri invece che siano intrinsecamente ostili alla loro esistenza e che, quindi, tendano ad ostacolarne l'azione.

Per quanto riguarda le tematiche, cioè l'oggetto dell'intervento di policy, non sembra di poter riconoscere differenze significative dovute al settore d'intervento: come si diceva all'inizio di questa nota, in letteratura è possibile rintracciare casi in 
campi assai disparati. Ciò nonostante, le questioni di routine, le pratiche distributive, i temi a basso livello di complessità, i problemi con una salienza collettiva contenuta, non necessitano di alcun tipo di azione imprenditoriale proprio perché l'innovazione richiesta è praticamente assente. Al contrario, le questioni più intrattabili e complesse, quelle minacciate dallo stallo, paralizzate da veti incrociati, caratterizzate da ambiguità nelle tecnologie applicabili, da contrastanti definizioni del problema di policy e da sostegno fluttuante, sono forse i puzzle che più richiedono l'eclettismo mostrato dai policy entrepreneurs. In tali circostanze, il fallimento degli approcci più consolidati sollecita soluzioni più innovative del semplice cambiamento al margine: ridisegnare le mappe cognitive con cui gli attori decisionali si confrontano, e inventare nuove alleanze diviene forse l'unico modo per sfuggire alla società a somma zero di Thurow $(1981)^{11}$.

Se i caratteri del tema affrontato, più che il suo settore di appartenenza, limitano i margini di libertà degli imprenditori di policy, così anche le variabili contestuali sembrano condizionarne l'operato. Su questo punto la letteratura non fornisce particolare evidenza empirica, mancando estese analisi comparative in materia. Si può comunque ipotizzare che, a parità di altre condizioni, laddove le dinamiche di policy-making appaiono maggiormente istituzionalizzate, cioè laddove esse si svolgono in strutture appositamente preposte, auto-referenziali e sostanzialmente poco permeabili rispetto alle manipolazioni messe in atto dagli stessi attori decisionali, il ruolo giocato dagli imprenditori di policy sia tendenzialmente marginale. Viceversa, percorsi decisionali poco definiti, privi di un referente istituzionale stabile e di apparati capaci di stabilizzare le interazioni, risultano più facilmente aperti (e vulnerabili) ad azioni imprenditoria$\mathrm{li}^{12}$. Ancora una volta, un esempio proveniente dal campo delle

11 Un buon esempio di come, a parità di altre condizioni, vi siano questioni che richiedono maggiore imprenditorialità d'azione - cioè capacità di innovare nelle soluzioni, nella costruzione del consenso, nell'argomentazione pubblica e nella legittimazione simbolica - è rappresentato dalla difficoltà di imporre politiche sottrattive in un settore largamente consolidato quale il welfare state: la loro probabilità di infrangersi contro le prime difese dello status quo è infatti tanto più alta quanto più usurati sono gli strumenti e le parole d'ordine utilizzate (cfr. Ferrera 1998).

12 Il citato lavoro di Dyson e Featherstone (1997) sul ruolo esercitato da Delors nel lanciare il progetto di Unione economica e monetaria fornisce una riprova a questa osservazione: l'istituzionalizzazione del processo derivante dall'apertura della Conferenza intergovernativa dedicata all'Uem diede nuova rilevanza agli apparati amministrativi 
politiche ambientali può chiarire tale divaricazione. A detta di molti osservatori, e secondo diversi indicatori empirici, l'Italia presenta un basso grado di istituzionalizzazione della politica ambientale: il ministero dell'Ambiente è un soggetto istituzionale che ha solo una decina d'anni di anzianità, mentre l'Agenzia nazionale è praticamente appena sorta; i rapporti sullo stato dell'ambiente sono pubblicati ancora irregolarmente, le leggi quadro dei diversi sotto-settori della policy ambientale sono state approvate in ritardo rispetto alla normativa di altri paesi, e la loro messa in opera è ancora eccessivamente incerta e a macchia di leopardo. In tale contesto, non stupisce trovare sia a livello centrale che a livello locale comportamenti imprenditoriali che in parte suppliscono e in parte si avvantaggiano dell'assenza di un circuito istituzionale stabilizzato. Viceversa, in Svezia, dove la difesa della natura ha una tradizione venerabile, dove gli apparati e gli interventi a protezione dell'ambiente vantano un lungo passato, dove l'internalizzazione delle proposte innovative così come la consuetudine corporativa permea l'azione delle istituzioni anche in questo settore d'intervento, lo spazio per imprenditori di policy è alquanto limitato (Giuliani, Rinkevicius e Sverrisson 1998$)^{13}$.

La stessa letteratura politologica sembra rappresentare una riprova indiretta di quanto appena sostenuto sulla base di una evidenza empirica ristretta. Gli studi sugli imprenditori di policy si concentrano nei paesi con sistemi decisionali che, per semplificare, potremmo definire maggiormente «pluralisti», quali quelli statunitense e britannico. Al contrario, laddove le istituzioni - tutte le istituzioni, e cioè il governo, il parlamento, l'amministrazione pubblica, i partiti, i gruppi corporativi, ecc. presentano una presa maggiore sul policy-making, questo tipo di soggetto decisionale è relativamente meno presente negli studi di policy. L'étatisme francese, con il monopolio esercitato dai suoi corpi burocratici, il corporativismo scandinavo, con la sua

e diplomatici, riducendo i margini di libertà per modalità d'azione meno tradizionali: «quando un tema è preso in mano dalle burocrazie, un singolo individuo non riesce più a padroneggiarlo» (intervista; ibidem).

13 Nello stesso lavoro si osserva come, al recente mutare del contesto in entrambi i paesi, con una graduale istituzionalizzazione dell'intervento ambientale in Italia e una certa deistituzionalizzazione dello stesso in Svezia (dovuta al fallimento dei tradizionali strumenti regolativi), sembra corrispondere anche una certa uniformazione dello spazio a disposizione degli imprenditori di policy: relativamente più ridotto in Italia e maggiore che in passato in Svezia. 
capacità di assorbire le tensioni e i conflitti riportandoli nella sfera interna delle decisioni collettivizzate, il Parteienstaat tedesco, con il ruolo accentratore esercitato dal cancelliere, non appaiono come i contesti istituzionali più favorevoli alla sua azione. Tuttavia non ne sono certo immuni. Per quanto riguarda l'Italia, un'interpretazione oramai diffusa ha accolto l'idea che il cosiddetto governo di partito abbia sostanzialmente lasciato poco presidiate le arene del policy-making; dal canto loro, sia le élites amministrative che quelle economiche non sono certo candidate ideali per un'irregimentazione dei processi decisionali; infine, governo e parlamento sono stati da tempo etichettati come istituzioni permeabili, incapaci di ridurre autoritativamente i margini per la definizione dei contenuti delle politiche pubbliche. Basta questo per avanzare l'idea che il policy-making italiano sia popolato da attori con capacità imprenditoriali? Probabilmente no, ma queste ci sembrano comunque buone ragioni per investigare con maggiore attenzione il punto di intersezione fra gli aspetti cognitivi e quelli strategici della produzione di politiche nel nostro paese.

Qualunque sia il potenziale per l'azione di policy entrepreneurs assicurato dal contesto nazionale, se non sarà la complessità crescente delle materie oggetto di intervento pubblico a sollecitarne implicitamente l'iniziativa, potranno forse essere i processi di internazionalizzazione a favorire la crescita di tale ruolo. Essi tendono nei fatti a premiare i soggetti decisionali capaci di muoversi su più terreni, di collocarsi negli snodi delle appropriate reti di policy, di padroneggiare le informazioni e gli stimoli provenienti da realtà nazionali diverse, di far tesoro delle esperienze già acquisite e di imitarle adattandole al contesto: in sintesi, essi tendono a premiare attori e istituzioni capaci di interagire e capaci di innovare. Insieme ad altre trasformazioni nelle modalità del policy-making, la perdita di rilevanza dei confini nazionali dovuta alle nuove tecnologie, alla condivisione di sovranità nelle organizzazioni internazionali più efficaci e, in generale, ai processi di globalizzazione, porterà probabilmente anche una nuova imprenditorialità nella produzione di politiche pubbliche. 
Riferimenti bibliografici

Capano, G. e M. Giuliani (a cura di) (1996), Dizionario di politiche pubbliche, Roma, La Nuova Italia Scientifica.

Dente, B. (1996), Tempo, in Capano e Giuliani (1996), pp. 404-409.

Dyson, K. e K. Featherstone (1997), Jacques Delors and the Re-launch of Economic and Monetary Union, relazione presentata al convegno Ecsa, Seattle, 29 maggio- $1^{\circ}$ giugno.

Fernandez, R.M. e R.V. Gould (1994), A Dilemma of State Power: Brokerage and Influence in the National Health Policy Domain, in «American Journal of Sociology», n. 6, pp. 1455-1491.

Ferrera, M. (1998), Le trappole del welfare, Bologna, Il Mulino.

Garrett, G. e B.R. Weingast (1993), Ideas, Interests, and Institutions: Constructing the European Community's Internal Market, in J. Goldstein e R.O. Keohane (a cura di), Ideas and Foreign Policy. Beliefs, Institutions, and Political Change, Ithaca, Cornell University Press, pp. 173-206.

Giuliani, M., L. Rinkevicius e A. Sverrisson (1998), Green Policy Entrepreneurship and Science and Technology Policy, in A. Jamison, (titolo da definirsi), Aarlborg, Aarlborg University Press, in corso di pubblicazione.

Gould, R.V. e R.M. Fernandez (1989), Structures of Mediation: A Formal Approach to Brokerage in Transaction Networks, in «Sociological Methodology», vol. 19, pp. 89-126.

Hajer, M.A. (1995), The Politics of Environmental Discourse, Oxford, Oxford University Press.

Heclo, H. (1974), Issue Network and the Executive Establishment, in A. King, (a cura di), The New American Political System, Washington, American Entreprise Institute, pp. 87-124.

Heclo, H. e A. Wildavsky (1981), The Private Government of Public Money, London, Macmillan.

King, P.J. e N.C. Roberts (1987), Policy Entrepreneurs: Catalysts for Policy Innovation, in «Journal of State Government», n. 1, pp. 172-179.

Kingdon, J.W. (1984), Agendas, Alternatives, and Public Policies, Boston, Little Brown \& Co.

Laffan, B. (1997), From Policy Entrepreneur to Policy Manager: the Challenge Facing the European Commission, in «Journal of European Public Policy», n. 3, pp. 422-438.

Ludlow, P. (1991), The European Commission, in R.O. Keohane e S. Hoffmann (a cura di), The New European Community. Decisionmaking and Institutional Change, Boulder, Westview Press, pp. $85-132$.

Majone, G. (1989), Evidence, Argument and Persuasion, New Haven, Yale University Press. 
Maraffi, M. (1996), Imprenditore di policy, in Capano e Giuliani (1996), pp. 188-192.

March, J.G. e J.P. Olsen (1997), Governare la democrazia, Bologna, Il Mulino.

Marsden, P.V. (1982), Brokerage Behavior in Restricted Exchange Networks, in P.V. Marsden e N. Lin (a cura di), Social Structure and Network Analysis, London, Sage.

Milward, H.B. (1980), Policy Entrepreneurship and Bureaucratic Demand Creation, in H.M. Ingram e D.E. Mann (a cura di), Why Policies Succeed or Fail, London, Sage, pp. 255-277.

Mintrom, M. (1997), Policy Entrepreneurs and the Diffusion of Innovation, in «American Journal of Political Science», n. 3, pp. 738770.

Mintrom, M. e Vergari, S. (1996), Advocacy Coalitions, Policy Entrepreneurs, and Policy Change, n. 3, pp.420-434.

Nugent, N. (1995), The Leadership Capacity of the European Commission, in «Journal of European Public Policy», n. 4, pp. 603-623.

Radaelli, C. (1998), Where Does Power Come From? The Narrative of Tax Competition in the European Union, relazione presentata al convegno Ecpr, Warwick, 23-28 marzo.

Regonini, G. (1995), Politiche pubbliche e potere, in G. Regonini (a cura di), Politiche pubbliche e democrazia, Napoli, Edizioni Scientifiche Italiane, pp. 21-102.

Regonini, G. e M. Giuliani (1994), Italie: au-delà d'une démocratie consensuelle?, in B. Jobert (a cura di), Le tournant néo-libéral en Europe, Paris, L'Harmattan, pp. 123-199.

Riker, W.H. (1983), Political Theory and the Art of Heresthetics, in A.W. Finifter (a cura di), Political Science: The State of the Discipline, Washington, The American Political Science Association, pp. 47-67.

Roberts, N.C. (1992), Public Entrepreneurship and Innovation, in «Policy Studies Review», n. 1, pp. 55-74.

Roe, E. (1994), Narrative Policy Analysis. Theory and Practice, Durham, Duke University Press.

Schattschneider, E.E. (1960), The Semisovereign People, New York, Rinehart Winston.

Schneider, M. e P. Teske (1992), Toward a Theory of the Political Entrepreneur. Evidence from Local Government, in «American Political Science Review», n. 3, pp. 737-747.

Schön, D.A. (1971), Beyond the Stable State, New York, Random House.

Schön, D.A. e M. Rein (1994), Frame Reflection. Toward the Resolution of Intractable Policy Controversies, New York, Basic Books.

Stone, D. (1988), Policy Paradox and Political Reason, Glenview, Scott Foresman.

- (1998), Think Tanks in the World, Manchester, Manchester University Press, in corso di pubblicazione. 
Thurow, L.C. (1981), La società a somma zero, Bologna, Il Mulino.

Wallis, J. e B. Dollery (1997), Autonomous Policy Leadership: Steering a Policy Process in the Direction of a Policy Quest, in «Governance: An International Journal of Policy and Administration», n. 1, pp. 1-22.

Weissert, C.S. (1991), Policy Entrepreneurs, Policy Opportunists, and Legislative Effectiveness, in «American Politics Quarterly», n. 2, pp. 262-274.

White, L.G. (1994), Policy Analysis as Discourse, in «Journal of Policy Analysis and Management», n. 3, pp. 506-525.

Wilson, J.Q. (a cura di) (1980), The Politics of Regulation, New York, Basic Books. 